\section{PEMBROLIZUMAB AND BEVACIZUMAB IN PLATINUM RESISTANT EPITHELIAL OVARIAN CANCER PATIENTS}

'Judith Michels*, ${ }^{2}$ Jean-Sebastien Frenel, ${ }^{3}$ Catherine Genestie, ${ }^{4}$ François Ghiringhelli, ${ }^{3}$ Caroline Brard, ${ }^{5}$ Benoit You, ${ }^{6}$ Anne Floquet, ${ }^{7}$ Lauriane Eberst, ${ }^{1}$ Rastilav Bahleda, ${ }^{1}$ Corinne Balleyguier, ${ }^{1}$ Angelo Paci, ${ }^{8}$ Joseph Ciccolini, ${ }^{1}$ Emeline Colomba, ${ }^{1}$ Fanny Pommeret, ${ }^{3}$ Christophe Massard, ${ }^{1}$ Patricia Pautier, ${ }^{1}$ Aurelien Marabelle, ${ }^{1}$ Alexandra Leary. ${ }^{1}$ Gustave Roussy Institute, Paris, France; ${ }^{2}$ Institut de Cancérologie de I'Ouest, Saint-Herblain, France; ${ }^{3}$ Gustave Roussy, Villejuif, France; ${ }^{4}$ Centre Georges François Leclerc, Dijon, France; ${ }^{5}$ nstitut de Cancérologie des Hospices Ci, Lyon, France; ${ }^{6}$ Institut Bergonié, Bordeaux, France; ${ }^{7}$ Institut de Cancérologie de Strasbourg, Strasbourg, France; ${ }^{8}$ La Timone University Hospital, Marseille, France

Background There is a medical need in platinum resistant ovarian cancer patients. Median progression-free survival (PFS) is 3.4 months with chemotherapy and 6.7 months with chemotherapy-bevacizumab combination regimens. ${ }^{1}$ RECIST overall response rate (ORR) is $11.8 \%$ and $27.3 \%$, respectively. The ORR is $15.9 \%$ for bevacizumab as a monotherapy with a median PFS of 4.4 months. ${ }^{2}$

Methods NCT03596281 An open-label phase 1b trial with a modified toxicity probability interval design to evaluate the combination of a flat dose of $400 \mathrm{mg}$ bevacizumab for 6 cycles and $200 \mathrm{mg}$ pembrolizumab until disease progression, unacceptable toxicity or completed 24 months of treatment in patients with platinum resistant ovarian cancer. The primary evaluation criteria is safety, the secondary endpoint is the efficacy.

Results 19 patients have been enrolled between January 2019 and February 2021 in 6 French centers. Patients characteristics are reported (table 1). No dose limiting toxicities were observed. Grade 3 treatment related adverse events occurred in 3 patients (i.e. arterial thromboembolism, bowel perforation, proteinuria and sepsis). No grade $4 / 5$ toxicities were induced. A median of 7 cycles (range 3-14) were administered. Median follow-up of patients was 4.1 months (1.8-23). The RECIST ORR was $26.3 \%$ (1 complete response and 4 partial responses) (table 2). The disease control rate was $78.9 \%$. The time to progression was not yet reached in 6 patients. The ORR was equivalent whether patients have been pretreated or not with bevacizumab (27.3 and 25\% respectively) (table 3). The ORR according to the combined positive score (CPS) for the evaluation of PD-L1 was $50.0 \%$ for CPS $\geq 10 \%(n=4), 30.0 \%$ for a CPS $\geq 1 \%(n=10)$ and $25.0 \%$ for $\mathrm{CPS}<1 \quad(\mathrm{n}=8)$ (table 4$)$.

Conclusions A chemotherapy-free regimen combining pembrolizumab and bevacizumab was well tolerated and showed encouraging results in heavily pretreated platinum resistant ovarian cancer patients independent of their previous challenge with antiangiogenic agents.

Acknowledgements Funding for this research was provided by Fondation Cancer du Luxembourg and Merck Sharp \& Dohme Corp., a subsidiary of Merck \& Co., Inc., Kenilworth, NJ, USA.

Trial Registration NCT03596281

\section{REFERENCES}

1.. Pujade-Lauraine $E$, et al. Bevacizumab combined with chemotherapy for platinumresistant recurrent ovarian cancer: The AURELIA open-label randomized phase III trial. J Clin Onco Off J Am Soc Clin Oncol 32,1302-1308 (2014).

2. . Cannistra SA, et al. Phase II study of bevacizumab in patients with platinumresistant ovarian cancer or peritoneal serous cancer. I Clin Oncol Off J Am Soc Clin Oncol 33,5180-5186 (2007).

Ethics Approval This study was approved by CPP Sud Méditerranée V institution's Ethics Board; approval number 18.020 (EudraCT number 2017-004197-34).
Consent Written informed consent was obtained from the patient for publication of this abstract and any accompanying images. A copy of the written consent is available for review by the Editor of this journal.

http://dx.doi.org/10.1136/jitc-2021-SITC2021.355 